\title{
The Negative Consequences of the Restructuring of the Albanian Banking System since the 90s
}

\author{
Prof. Ass. Dr. Alqi Naqellari \\ Mediterranean University of Albania \\ Ismail Qemali University of Vlore \\ alqinaqellari@gmail.com
}

\section{Doi:10.5901/mjss.2013.v4n6p435}

\begin{abstract}
In this paper we focus primarily on an analysis of the economic consequences of the reorganization of the banking system after 1990, starting with the economic reality of Albania. The Albanian economy as an economy in developing countries should have a growth and significant improvements on all macroeconomic indicators. Nowadays indicators in relation to the year 2009, the year in which they were shown publicly the consequences of the global recession these show that the Albanian economy continues to worsen. This deterioration was influenced by the Albanian government fiscal policies implemented in recent years. In this deterioration affects also the Bank of Albania, the structure and functioning of the banking system in general. Precisely in this regard will try to answer the question: Why the Bank of Albania cannot control inflation which is also its main objective? For this it will list the main factors that determine inflation in Albania. We will explain why it should not be given credit and accept deposits in foreign currency by commercial banks. The currency should not be concentrated in the Central Bank as its monopoly? We will explain why it was not necessary privatization of second-tier banks. By answering these questions we will also examine the negative consequences of this restructuring of the Albanian economy.
\end{abstract}

Keywords: Total privatization, monetary policy, exchange rate, oligopoly, the commercial bank owned by the state.

\section{Introduzione}

Dopo il 1990, in Albania è iniziato il processo di privatizzazione dell'economia per trasformare l'economia da un'economia in cui sui fattori economici esisteva solo la proprietà statale in un'economia di mercato basata sulla proprietà privata, sulla concorrenza e la libera iniziativa. Profondi cambiamenti nell'economia erano da eseguire nel campo della proprietà privata. La privatizzazione delle imprese statali di servizi e delle imprese per l'estrazione progredivano rapidamente. Questo processo è stato accompagnato da cambiamenti nell'organizzazione del sistema bancario. Questi settori dovevano camminare parallelamente al fine di non compromettere l'un l'altro nel progresso economico. In questo modo le riforme della proprietà privata si sono diffuse nel sistema bancario. In questa analisi ci concentreremo sul sistema bancario ed esamineremo l'efficacia della riorganizzazione del sistema bancario.

\section{La dinamica del sistema bancario Albanese prima e dopo il 1990}

Il sistema bancario albanse prima del 1990 è stato un sistema di un livello. Nel centro del sistema era la Banca d'Albania, dalla quale erano completamente dipendenti la banca Nazionale Commerciale, la Banca Agricola Commerciale e l'Agenzia di Risparmio la quale dopo il 1997 si trasformò in Cassa di Risparmio. La loro attività era comune. Questo sistema bancario era adatto al sistema economico centralizzato in atto in Albania. Gli strumenti politici ed economici diretti messi in atto dalla Banca d'Albania erano: il limite di credito per le banche sotto la sua supervisione, il tasso massimo di interesse sui depositi e il tasso minimo di interesse sui prestiti. In questo modo il suo principale obiettivo era la crescita economica. Questo era l'obiettivo fino al 1990, perché fino a quel tempo non era noto il concetto dell' inflazione e della disoccupazione. Dopo il 1990 la situazione è cambiata. L'ingresso dell'Albania nella economia di mercato, il cambiamento del rapporto della proprietà è stato associato a fenomeni tipici di questo sistema come l'inflazione e la disoccupazione.

Per adattarsi meglio alla situazione creata, il 23.12.1997 è stato approvato la legge n. 8269, "La Banca d'Albania". Ai sensi dell'articolo n. 16 della legge n. 7491, il 29.4.1991 "Sulle principali disposizioni costituzionali", questa legge è stata modificata dalla legge n.8417, il 21.10.1998. II sistema bancario Albanese è stato sudiviso in due livelli. Nel primo livello è presente solo la Banca d'Albania con i suoi obiettivi e funzioni, ed al secondo livello sono comprense tutte le 
banche commerciali che non dipendono dalla Banca d'Albania per le loro politiche interne per la realizzazione dei loro prodotti. In questoperiodo in Albania erano presenti solo banche commerciali. In questo modo c'è stato il passaggio graduale verso l'attuazione di tre strumenti politici ed economici indiretti. 1. Le operazioni sul mercato aperto. Queste azioni riguardano la vendita o l'acquisto di attività finanziarie. 2. Determinazione della riserva obbligatoria. Ė stato determinato il $10 \%$ dei depositi. 3 . II tasso di interesse di base sulle operazioni pronti contro termine (repo). Lo scopo dell'attuazione dei nuovi strumenti è stato la realizzazione dell'obiettivo principale della Banca d'Albania. Dopo il 2000, quando in Albania non esisteva nessuna banca commerciale, gli strumenti indiretti hanno trovato piena attuazione. Questo è accaduto perché fino ad allora c'erano sia banche con capitale sociale interamente statale che a capitale interamente privato.

Nel $1^{\circ}$ capitolo, l' articolo n.3 della legge del 1997 afferma, tra l'altro:

1. II principale obiettivo della Banca d'Albania è quello di raggiungere e mantenere la stabilità dei prezzi. I principali compiti della Banca d'Albania sono i seguenti:

a) Sviluppare, adottare ed attuare la politica monetaria della Repubblica dell'Albania, in conformità con il suo obiettivo primario .

La Banca d'Albania ha avuto ed ha come obiettivo principale, presente e futuro, quello del "raggiungimento ed il mantenimento della stabilità dei prezzi". Per quanto riguarda gli altri obiettivi macroeconomici fondamentali quali la crescita del PIL e la diminuzione del tasso di disoccupazione al fine di raggiungere un livello naturale, non ha avuto alcun obiettivo concreto, anzi si sono invece visti come obiettivi di secondo o di terzo livello, dando una particolare attenzione alla stabilità finanziaria.

La Banca d'Albania non si è mai ritenuta responsabile per la realizzazione di questi obiettivi, concentrandosi soltanto nel fornire pareri per adattarsi alla situazione. Nei primi mesi del 2013, quando gli indicatori macroeconomici, come espressione di fenomeni economici, sono peggiorati ha dichiarato il fallimento della politica economica.

Gli obiettivi nel contesto di indicatori macroeconomici sono stati e rimangono gli obiettivi del governo Albanese. II motivo per cui questa banca non ha tali obiettivi è che ha preso in modo stereotipato gli obiettivi della Banca Centrale Europea. II problema per questi paesi, in queste condizioni, è il tasso di inflazione. In Albania, il problema principale non è raggiungere e mantenere il livello dei prezzi, ma è aumentare il prodotto interno lordo a ritmi più alti e ridurre il tasso di disoccupazione. Questo perché l'economia albanese è un'economia in fase di crescita e non nel potenziale prodotto. Le economie simili all'economia albanese non possono svilupparsi senza aumentare il tasso di inflazione. Assicurare la crescita economica nelle condizioni in cui si trova l'albania, avendocome obiettivo quello di frenare l'inflazione, è un controsenso. Frenare l'inflazione porta ad un ulteriore aumento del tasso di disoccupazione e non il suo declino. L'inflazione nelle condizioni del'albania e sinonimo di crescita economica.

La Banca d'Albania controlla l'inflazione. Si tratta semplicemente di un influenza frivola, perché l'inflazione in generale è determinata dall'inflazione importata e da altri fattori che non sono sotto il suo controllo.

\section{I fattori che influenzano l'inflazione nell'economia albanese e da chi vengono influenzati questi fattori?}

\subsection{L'economia albanese è una piccola economia aperta.}

II tasso di apertura è del 42,2\% (esportazioni + importazioni 544,003 $\mathrm{ml} / 1.282 .254 \mathrm{ml} \mathrm{PIL}$ ). In questo paese non corrispondono le entrate con le spese. I tassi di interesse applicati dipendono daii tassi di interesse applicati da altri paesi con grandi economie aperte e principalmente dal tasso di interesse della Banca Centrale Europea e dalla Federal Reserve degli Stati Uniti perchè il dollaro e l'euro circolano liberamente e occupano una parte visibile del mercato monetario albanese. La sua politica monetaria dipende dalla politica monetaria delle banche centrali di altri paesi con una grande economia aperta. Dopo il 2008, con l'inizio del 2009, ha dovuto adattarsi alla recessione nei paesi europei e non essere dell'opinione che la nostra economia non sarà influenzato da questa recessione. Nell'economia albanese non avrebbe avuto effetto la trasmisione per mancanza di capitale finanziario e di altre istituzioni finanziarie, e non perchè non sarebbe influenzata dal declino dell'Europa e dell'America. L'impatto positivo o negativo della recessione globale è un altro problema. II passare del tempo ha dimostrato che la recessione globale ha influenzato e continua ad influenzare l'economia attuale albanese.

Per adeguarsi ai fenomeni mondiali la Banca d'Albania all'inizio dei cambiamenti del tasso d'interesse della Banca Centrale Europea, ha dovuto ridurre il tasso di interesse a ritmi più elevati, al fine di influenzare in modo significativo sui

${ }^{1}$ Queste cifre sono tratte dal Rapporto statistico febbraio 2013. Banca d'Albania. 
tassi di interesse che dovevano essere applicati sui prestiti in lek, dalle banche di secondo livello. In questo modo la moneta Lek sarebbe stata in concorrenza con l'euro e il dollaro in condizioni di parità. In questi anni, la concorrenza tra loro è stata sleale perché il Fed e la BCE hanno abbassato il tasso fino a 0,5\% in un momento in cui la Banca d'Albania I'ha teneva a 6,25\% (2009) e nel 2013 l'ha abbassato al 3,75\%. Con una politica espansiva la Banca d'Albania stimolerà la crescita della domanda aggregata, che a sua volta influenzerà la crescita dell'offerta. L'effetto reale di questa azione sarebbe una reale crescita economica e l'apprezzamento del ruolo della moneta albanese Lek in questa crescita. Con l'attuazione di una politica non di sostegno alle politiche della Banca Centrale Europea e la Federal Reserve, la Banca d'Albania ha contribuito ben poco all' aumento o alla diminuzione della base monetaria e il tasso di inflazione.

\subsection{I prodotti agricoli non soddisfano le esigenze della popolazione urbana.}

In Albania ci sono circa 350 mila aziende agricole e zootecniche. Circa il 94,7\% di loro commercializzano e vendono direttamente sul mercato piccole quantità di prodotti. La produzione interna non soddisfa i bisogni della popolazione urbana. Le ragioni per cui è diminuita la produzione agricola:

- La diminuzione della superficie del terreno agricolo da 579.000 ettari nel 1990², a 402.000 ettari nel 2009. Nel 1986, 203 mila ettari erano piantati di grano e nel 2009 sono stati piantati solo 82 mila ettari. Rispetto al 1990, nel 2009 sono stati piantati considerevoli superfici di foraggera, mentre tutte le altre culture avevano diminuito la superficie coltivata.

- II grado di meccanizzazione è molto basso rispetto a paesi con i quali I'Albania ha rapporti commerciali. II parco della meccanica in Albania ha solo 7883 trattori con pneumatici, 3064 macchine seminatrici e 3893 coltivatori. In Albania, 199531 sono le aziende agricole che lavorano a mano.

- La quantità di fertilizzanti chimici per ettaro è bassa. Nel 2009 sono stati utilizzati 118 mila tonnellate di fertilizzanti azotati e fosfati, rispetto a 330 mila tonnellate che erano state utilizzate nel 1990.

- La superficie delle serre è di 728 ettari di cui solo 55 ettari sono coperte con vetro. Questa area è molto piccola rispetto alle esigenze della popolazione nel suo complesso.

- I prodotti agricoli del paese, escludendo i periodi di punta, in qualsiasi periodo non determinano i prezzi di mercato.

- Le esportazioni di prodotti agricoli sono molto bassi rispetto alle importazioni. Così nel 2009 il rapporto era di 1/9 (una esportazione su 9 importi). I rapporti per settore sono stati:

\begin{tabular}{lccl}
\multicolumn{1}{c}{ Settore } & \multicolumn{2}{c}{${\text { Anno } 2009 \text { milioni lek }^{3}}$} \\
& Esportazioni & Importazioni & Percentuale \\
Agricoltura & 2.630 & 17.315 & $1 / 6.5$ \\
Alevamento & 964 & 9.300 & $1 / 9.6$ \\
Trasformazione alimentare & 3.942 & 42.602 & $1 / 10.8$ \\
Totale & 7.536 & 69.217 & $1 / 9$
\end{tabular}

Le esportazioni $=$ Agricoltura . Le principali esportazioni agricole sono le piante medicinali con il $57,3 \%$, le verdure e cocomeri con l' $11 \%$, la frutta con il $6,6 \%$, le patate con l' $1,58 \%$ ecc. La quantità di frutta e verdura esportata è bassa con 14352 tonnellate di verdure, 333 tonnellate di patate e 1958 tonnellate di frutta.

= Alevamento. La maggior parte delle esportazioni di prodotti di origine animale sono le interiora degli animali con il $72 \%$ e le uova con il $21 \%$. Gli animali vivi con il $5,2 \%$.

$=$ Trasformazione Alimentare. La principale esportazione di alimenti trasformati è il pesce lavorato che occupa il $60 \%$, il cuoio naturale con il $17,5 \%$, acqua minerale e con il $7,4 \%$, il tabacco con il $6,6 \%$, ecc.

Le importazioni. $=$ Agricoltura. Le verdure importate sono di 17819 tonnellate, di cui 4.760 tonnellate di legumi secchi per un valore complessivo di 1.255 milioni 0 il 7,2\% del totale. Pari a 4,4 volte superiore al valore delle esportazioni. Le patate importate sono di 20.048 tonnellate, 380 tonnellate di fagioli, 77832 tonnellate di frutta, ecc. Vengono importate 267256 tonnellate di frumento, 63855 tonnellate di granturco e 30810 tonnellate di farina di

\footnotetext{
2 I dati utilizzati per il 1990 e prima, sono tratte da "l'annuario statistico della Albania. (Ministero dell'Economia Dipartimento di Statistica) del 1991".

3 I dati utilizzati nel secondo paragrafo per il 2009 sono stati ottenuti dal Ministero delle Politiche Agricole Annuario statistico 2009, pubblicato nel mese di aprile 2010. Indirizzo Internet (Ministero delle Politiche Agricole Yearbook 2009 (pdf)).
} 
frumento. II valore totale delle importazioni di cereali occupa oltre il 40\%. Tale importo copre il $100 \%$ delle esigenze della popolazione urbana e una parte delle esigenze della poplazione rurale. II 100\% di riso, caffè, tè, spezie ecc, usato viene importato.

= Alevamento. Occupa oltre il 99\% delle importazioni di animali vivi e della carne.

= Trasformazione alimentare. Vengono importati 28 tipi di prodotti trasformati, come i derivati del latte, la carne, la farina, i grassi alimentari, la salsa e il succo di pomodoro, le sigarette, il cacao, la margarina, lo zucchero, l'olio vegetale, il cibo per gli animali ecc. Vengono importati notevoli quantità di pasta, salsicce, verdure in scatola, acqua minerale, birra, alcolici, vino, ecc.

Tutte le cifre riportate sopra mostrano chiaramente che la base di questi prezzi è il paese di origine. La conclusione è semplice, i prezzi vengono determinati nei paesi importatori. In Albania, il loro prezzo sarà più alto di quello dei paesi di origine a causa del regime fiscale adottato.

\subsection{Nel 2011 il rapporto dell'import-export è stato 1/3.}

Gran parte del paniere di beni sulla base dei quali è stato calcolato l'indice dei prezzi al consumo (CPI) sono stati importati. Sopra abbiamo presentato alcuni elementi del ramo dell'agricoltura che dimostrano che i prezzi di una decina di beni sono definiti nei paesi di origine e non in albania. Se facciamo riferimento ad altre sfere di produzione, allora possiamo dire che pochi prodotti si realizzano al $100 \%$ in Albania. La maggior parte di loro vengono importati. I loro prezzi influenzano il prezzo dei prodotti nazionali. Per questo facciamo riferimento al rapporto dell' import-export complessivo per il 2012 nelle voci principali4:

- Prodotti alimentari, bevande e tabacco 1/7,27 (12,8 esportazioni / importazioni 93,17)

- Minerali, combustibili ed energia 1/1.4 (76,1 esportazioni / importazioni 110.4)

- Chimica e plastica da 1/33 (2 esportazioni / importazioni 66)

- Cuoio e articoli derivati 1/3.6 (2.7 esportazioni / importazioni 9.7)

- Prodotti di Legno e carta 1/3.4 (5.3 esportazioni / importazioni 18.1)

- Prodotti tessili e scarpe 1/0.71 (62 esportazioni / importazioni 48.3)

- Macchine e attrezzature 1/12.8 (7.6 esportazioni / importazioni 97.7)

- Altro 1/4.3 (3.9 esportazioni / importazioni 16.8)

I dati mostrano che escludendo la voce dei prodotti tessili e le calzature in tutti gli altri voci predominano le importazioni. Anche in questa voce ci sono le riesportazioni che occupano una parte sostanziale di esso. Nel mercato delle calzature e dei vestiti dominano le merci straniere, piuttosto che la produzione nazionale. La produzione nazionale circola molto poco. La stessa cosa accade con il carburante. L'Albania produce circa 700 mila tonnellate di combustibile. La maggior parte di essa viene esportata. In questo modo il prezzo del mercato è determinato dal prezzo dei combustibili importati non dal prezzo del combustibile prodotto all'interno.

La conclusione è semplice, i prezzi dei beni di cui sopra sono definiti nei paesi produttori. La Banca d'Albania può influenzare poco o quasi per niente. II governo ha un influenza maggiore con la sua politica fiscale rispetto alla Banca Centrale con la sua politica monetaria.

\subsection{II tasso di cambio della moneta Albanese lek è flessibile.}

La Banca d'Albania interviene nel mercato comprando o vendendo valuta estera. Questo lo fa quando il Lek albanese si è deprezzato di più rispetto alle previsioni. I tassi di cambio influenzano in modo significativo il livello dei prezzi nel paese e l'economia del paese. Questo fenomeno risulta dalla dichiarazione di esportazione-importazione esposto sopra. Se si guarda ai tassi di cambio a partire dal 2009 fino ad oggi vedremo un aumento significativo dell'euro nei confronti del Lek albanese. Nel 2009-2012 abbiamo avuto i seguenti rapporti: 1/132.06, 1/137.79, 1/140.33 e oggi 1/141. I dati mostrano che il tasso di cambio ha avuto un impatto negativo sul livello dei prezzi. Si prega di notare che il deprezzamento del Lek albanese aumenta il prezzo delle importazioni. Quali sono i fattori che hanno contribuito alla svalutazione del lek albanese rispetto a valute estere? La domanda e l'offerta di moneta:

Dal lato della domanda,abbiamo questi fattori :

4 I dati sono stati ottenuti dal Rapporto statistico marzo 2013. Banca d'Albania, tab. 2.3. Esportazioni per gruppi di prodotti, in milioni di lek e tab.2.4, le importazioni per gruppi di prodotti in milioni di lek. 
a) La domanda di moneta per importare beni dall'estero, che ha un impatto negativo sulla valutazione di apprezzamento in quanto le importazioni sono aumentate. La crescita di questa domanda è normale perchè molte delle sua voci, come l'acquisizione di macchinari e manufatti che hanno un'impatto positivo sulla crescita dell'economia.

b) La domanda delle compagnie estere finanziarie bancarie e non, che operano nel territorio della Repubblica di Albania. In questo gruppo possiamo citare 13 Banche commerciali con capitale straniero che esportano al di fuori del territorio della Repubblica di Albania i loro profitti, non in lek ma in valuta estera. Loro esportano la valuta albanese, sotto forma di prestiti ai non residenti o attraverso le banche madri. Queste cifre secondo la stampa quotidiana superano i 200 - 300 milioni di euro all'anno.

c) La domanda delle compagnie non finanziarie estere come le società di telefonia mobile, AMC, Vodafone, Eagle,Plus ecc . Tutti i loro profitti queste società le delegano alle imprese madri che si trovano all'estero. Questa moneta loro se lo assicurano semplicemente comportandosi da suo compratore nel mercato. In questo modo aumentano la pressione sulla svalutazione del lek.

d) Le richieste delle aziende straniere che vincono gare d'appalto per la costruzione di opere strategiche nel territorio dell'Albania. In questo gruppo si comprendono le società turche, greche e macedoni che hanno intrapreso l'esecuzione di lavori di alcuni reti stradali e le loro rispettive gallerie. Altre società straniere hanno intrapreso la costruzione di centrali idroelettriche o centrali elettriche. Costantemente queste aziende fanno pressione negativa sul tasso di cambio del Lek albanese. Anche questi hanno un impatto negativo sul livello dei prezzi.

e) La domanda delle aziende che prendono in concessione opere strateghiche.

f) La domanda delle società straniere di produzione e delle società per azioni operanti nel territorio della Repubblica di Albania. Con queste si intendono le società greche (tessile, calzature, cemento, ecc), le società italiane (tessile, calzature, commercio, ecc), canadesi (petrolio), turchi (lavorazione dei minerali, commercio, ecc) che operano nel territorio della Repubblica di Albania. La loro influenza sul tasso di cambio è negativo, con conseguente aumento fittizio della domanda di valuta estera.

g) La domanda delle società albanesi o miste nel campo dell'edilizia. Queste aziende mettono le costruzioni finali in vendita, trattando i prezzi in euro $/ \mathrm{m}^{2}$ e non in leke/ $\mathrm{m}^{2}$. In questo modo loro fano si che aumenti la domanda dei cittadini di prestiti verso le banche commerciali. Questo è un fattore che aumenta la richiesta di credito in modo esponenziale e questo ha un'impatto negativo sul livello dei prezzi.

h) La domanda sulla vendita dei terreni 0 altri capitali in valuta.

i) Il pagamento di una parte del personale di aziende straniere che operano albania in valuta estera

j) La domanda degli uomini d'affari, dei ministri, dei parlamentari, ecc. per la valuta estera in modo da operare quando vanno all'estero. In questo modo queste persone portano all'estero questa valuta, per poter effettuare: = Servizi di Stato,

= Servizi e lavoro privato,

$=$ Depositi di valuta in banche straniere

$=$ Investimenti all'estero in Italia, Grecia, Macedonia, ecc

= Vacanze estive, invernali, ecc.

Dal lato dell'offerta abbiamo questi fattori:

a) La maggior parte dei flussi in entrata della valuta nell'economia albanese non avviene dall'esportazione delle risorse 0 di prodotti diversi, ma una parte significativa proviene dalle risorse umane esportate, cioè da profughi ed emigranti albanesi che lavorano in altri paesi. Accanto ad essa ci sono anche le entrate provenienti dal settore informale.

In particolare se si confronta il bilancio dei pagamenti (BT) con il Saldo di Conto Corrente (NA) si nota esattamente questa differenza. Nel 2010, il BT $=-2,082,7 \mathrm{ml}$ di Euro, NA $=-1,018.6$ miliardi di euro. Nel 2011, BT = -2,241.6 miliardi di euro, NA = -1,185.4 miliardi di euro. Nel 2012, BT = -1,999.2 ml di euro e NA = $-1,021.3 \mathrm{ml}$ in euro. Chiaramente la maggior parte delle importazioni si chiude attraverso altri account.

b) I trasferimenti dai privati per gli anni $2010-2012$ sono stati $877,1 \mathrm{ml}$ di euro $877,6 \mathrm{ml}$ di euro e $846,7 \mathrm{ml} \mathrm{di}$ euro. La voce comprende anche le rimesse che hanno avuto un impatto positivo sulla valutazione del Lek albanese, e quindi anche sul livello dei prezzi. 
c) I servizi in entrata per il triennio 2010-2012 sono stati, 1.750,7 miliardi / euro, 1,747.4 ml / euro e 1, 655,1 $\mathrm{ml} /$ euro. All'uscita sono stati $-1,518.9 \mathrm{ml} /$ euro, $-1,612.4 \mathrm{ml} /$ euro e $-1,459.9 \mathrm{ml} / \mathrm{eur0}{ }^{5}$. Anche se il reddito di tale voce è molto elevata e vale la pena di notare che i costi sono altrettanto elevati. In questo modo il suo bilacio ha un reddito basso. Questo per me è la voce più discussa per controllare ed astenersi dall'abuso delle riserve in valuta estera. Questo include tutte le voci del punto "j" le quali aumentano la domanda di valuta estera.

d) Negli anni il reddito è rimasto negativo sul saldo del conto corrente.

e) II conto capitale e finanziario ha avuto un impatto positivo. Gli investimenti esteri diretti sono aumentati significativamente da 788,5 ml / euro nel 2010 in 715,5 ml / euro nel 2011 e 727,2 $\mathrm{ml} /$ euro nel 2012. II loro impatto è stato positivo nei confronti del Lek albanese e quindi anche sul livello dei prezzi. In tanti anni hanno chiuso il disavanzo delle partite correnti.

3.5 Nell'economia albanese sono presenti oltre le strutture del mercato quali la concorrenza monopolistica, monopolio e oligopolio, anche due poli, alleanze e collusioni.

Questi operano sotto il nome di associazioni e molto spesso si sono esposte sotto forma di pressione sul governo. Oggi insieme ad altri fattori determinano il livello dei prezzi sul mercato. Citiamo l'associazione dei costrutori, dei carburanti, del trasporto urbano, delle banche, la panetieri, dei dentisti, ecc.

Nel settore bancario, i tassi di interesse sui prestiti, a prescindere dalla politica che applica la Banca d'Albania, dipendono da loro. Con tutta la pressione che rende la Banca d'Albania alle banche commerciali per facilitare e aumentare i prestiti, le banche vedono la loro posizione strategica, senza prendere in considerazione le sue esigenze. In questo modo, le banche commerciali hanno minato il ruolo della Banca d'Albania a guidare l'offerta del mercato. Esse danno credito alla valuta che vogliono e al tasso di interesse deciso da loro.

\subsection{La produzione nazionale.}

Per quanto riguarda la produzione interna ed il suo impatto sul tasso di inflazione si può parlare con molte riserve dopo tutte le argomentazioni tecniche che sono sopra elencati. Così sono pochissime le merci prodotti in tali quantità da superare la metà del fabbisogno totale. Se questo accadesse allora il prezzo interno influenzerà il prezzo dell'importazione. Ciò si verifica solo nel breve termine nel settore agricolo, nel mese di giugno, luglio e agosto. Non accade in altri settori. Per quantificare la quantità ed il valore della produzione interna, ne parliamo con riserve, perché ci sono gravi carenze nel suo calcolo. Se consideriamo che la produzione agricola è misurata attraverso gli indagini. Nessuno può dire esattamente quanto è la produzione di prodotti agricoli in Albania perché si pesa solo il prodotto venduto, mentre il resto non viene quantificato. Se fosserò vere le cifre fornite dal Ministero delle Politiche Agricole, allora non avremmo bisogno per le importazioni, anzi ci sarebbe l'esporto quotidiano di prodotti agricoli.

\subsection{La stabilità politica e sociale del paese e dei paesi con i quali ci sono dei rapporti commerciali.}

La stabilità economica e politica dei paesi che hanno forti relazioni economiche, come l'Italia e la Grecia non sono stati soddisfacenti. Quindi, I'Italia e la Grecia non sono ancora fuori dalla recessione e dalla depressione. II Kosovo, la Macedonia o la regione se non hanno avuto problemi significativi politici ed economici hanno avuto problemi sulla loro stabilità interna. La Turchia, I'Ucraina, la Bielorussia, ecc., sono stati i paesi più stabili.

Sopra abbiamo descritto i fattori chiave che influenzano il tasso di inflazione e abbiamo mostrato la relazione della Banca d'Albania con esso.

Di seguito risponderemo alla domanda:

\section{Quali sono le principali ragioni che la Banca Centrale dell' Albania non può controllare l'inflazione?}

4.1 La circolazione di due valute estere come l'euro e il dollaro senza limitazioni, allo stesso modo della moneta albanese lek.

5 tab.2-1, Settore esterno, Bilancio dei pagamenti in euro. 
Euro è il nome della moneta dell'Unione europea (la zona euro), e dollaro è il nome della valuta degli Stati Uniti d'America e di alcuni altri paesi che lo utilizzano. All'interno del territorio albanese in qualsiasi banca commerciale si può aprire un deposito di risparmio in valuta nazionale, in euro e in dollaro, si può anche prendere un prestito in moneta nazionale, in euro e dollaro senza alcuna restrizione o limitazione. Non c'è nessun altro paese in Europa occidentale a consentire tale azione. In Europa, nella zona euro il prestito è concesso in euro, e in Inghilterra è dato in sterline. La valuta estera può essere acquistata nel apposito negozio di scambio (exchange). Anche i soggetti economici europei non sono interessati a prendere prestito 0 aprire depositi in una valuta estera.

Qualcuno dirà, è molto buono, perché un giorno ci sarà un passo nella zona euro e che il loro fatturato ha molti vantaggi. La mia risposta è che "la libera circolazione e l'uso di valuta per depositi e prestiti è un errore che dovrebbe essere eliminato immediatamente." Perché dico questo? Perché ci sono altre due conseguenze negative associate all'oggetto della nostra analisi oltre agli altri fattori che abbiamo evidenziato. "La Banca d'Albania non può controllare l'inflazione per motivo della mancanza di efficacia nel mercato monetario."

La Banca Centrale prepara, adotta ed attua il piano di politica monetaria. Questo significa che attraverso tre strumenti, le operazioni di mercato aperto, riserva obbligatoria e il tasso base di interesse (REPO), aumenta 0 diminuisce la quantità di moneta in circolazione, in funzione della situazione economica albanese. In questo modo si stimola l'emissione della moneta lek oppure il suo ritorno nelle banche. E lo fa solo per la moneta Lek avendo questi tre strumenti. II più importante di questi è il "tasso base di interesse" che altrimenti viene chiamato, tasso di sconto nel riacquisto di pronti contro termine. Abbassando questo tasso, aumenta l'interesse da parte delle banche commerciali di comprare di più, ma dall'altro canto quanto si riduce questo interesse si riduce anche il tasso di interesse sui depositi e di conseguenza l'interesse su di esse. La riduzione dei tassi di interesse stimolerà le imprese ad affrontare i prestiti in lek. In questo modo potranno diventare più attraenti e daranno un impulso allo sviluppo economico. Quindi, riducendo il tasso di interesse viene incoraggiato l'uscita di moneta al di fuori della banca quindi aumentare la domanda aggregata. Se solo avessimo lek in circolazione, avrebbe completato le sue funzioni, che sono, di scambio e strumento di pagamento, unità standard di conto, riserva di valore, unità standard per i pagamenti differiti. La prima funzione come mezzo di scambio e di pagamento, non è completa per l'assenza di una delle sue caratteristiche, qualeche pertanto deve essere accettato da tutti. In effetti non è accettato da tutti, perché una buona parte delle operazioni all'interno del territorio della Repubblica di Albania è effetuatto in euro e dollaro. Questo ha portato alle conseguenze che abbiamo detto e alle quali ci soffermeremo sotto.

In Albania allo stesso modo della moneta lek, che è la valuta ufficiale della Repubblica di Albania, ci sono in circolazione l' euro ed il dollaro. Queste due valute contano la maggior parte del totale dei crediti in generale e si stanno avvicinando al 50\% dei depositi. Alla fine del 2012 i depositi in valuta estera erano il 48,76\% (453830/930701), rispetto il $51,24 \%$ in valuta nazionale del totale dei depositi. I depositi in valuta estera tendono ad aumentare. Se oggi i depositi occupano il $51,24 \%$ del totale dei depositi sotto forma di prestiti vanno il $43,2 \%$ (206398/476871). Dall'altro canto se il $48,76 \%$ del totale dei depositi è in valuta estera, il $74,3 \%$ viene datto sotto forma di credito nel territorio dell'Albania. Se si includono i prestiti o depositi al di fuori del territorio dell'Albania queste cifre superiano il $100 \%$.

Visualizzando dei report sui depositi in valuta estera e in lek, e il rapporto dei prestiti sui depositi si vede chiaramente che il Lek albanese tende ad essere rimosso dalla circolazione e di fatto ad eliminare il suo impatto sulla crescita economica, incoraggiando gli investimenti delle imprese. II ruolo influente nella crescita degli investimenti delle imprese l'ha adottato l'euro e il dollaro, cioè le valute estere, in un momento in cui dovrebbe accadere il contrario. E 'a questo proposito che si è ridotto il ruolo della Banca Centrale della Repubblica di Albania, non realizzando o suoi compiti principali, citati sopra.

I depositi in lek occupano la quota maggiore di raccolta totale, perché hanno un tasso di interesse molto più alto dell'euro e del dollaro. In particolare nel 2012 abbiamo avuto questa situazione: depositi di 12 mesi hanno avuto un tasso di interesse del 5,38\%, in dollaro del 3,06\% e in euro del 2,56\%. I depositi di 24 mesi hanno avuto un tasso di interesse del $6,5 \%$, in dollaro del $2,56 \%$ e in euro del 3,8\%. Da questi dati i più preferibili sono i deposit in lek perché hanno tassi di interesse più elevati. Ed è proprio questo che ha portato allo sviluppo della raccolta in lek.

Per quanto riguarda la concessione di credito, come detto sopra i prestiti in valuta prevalgono e occupano il $62.05 \%$ del totale dei crediti, indipendentemente dalla loro raccolta che rappresenta solo il $48,76 \%$ del totale dei depositi. I tassi di interesse sui prestito in valuta estera sono state:

- da 6 mesi a 1 anno in lek, del 10,28\%, in euro del 6,92\% e in dollaro del 6,96\%

- da 1 anno a 3 anni in lek, del $14,35 \%$, in euro del 7,41\% e in dollaro del 6,38\%. Cosa significano questi dati? Questi dati indicano che i soggetti economici sono interessati ad ottenere prestiti in euro e non in Lek albanese perché la loro differenza è troppo grande. II prestito da 1-3 anni la differenza in euro è di $6,94 \%$. Se 
si fa riferimento ai prestiti sopra i 5 anni, che sono di solito preferibili perché le persone prendono più mutui per la casa, questi tassi hanno ridotto il divario tra di loro. In particolare il prestito di 5 anni ha un tasso di interesse in lek del 9,51\%, in euro del 7,01 e in dollaro del 5,52\%. Ma perché le persone si rivolgono ai prestiti in euro quando la differenza è solo di $2,5 \%$, e quando l'apprezzamento del lek è molto significativo ogni anno? La ragione è che i proprietari degli edifici li vendono in euro al metro quadrato e non in lek.

Perché esistono tutte queste differenze tra i tassi di interesse sui deposito e di prestito in valuta nazionale e in valuta estera? II motivo è tecnico. La politica monetaria della Banca Centrale d'albania per conto del lek albanese, della Banca Centrale Europea per l'euro e della Banca Centrale Americana (Fedi) per il dollaro.

La Banca Centrale Europea e il Fedi costantemente dal 2009, come l'anno in cui si sono visti gli effetti della recessione, hanno applicato una politica espansionistica potente. Fedi ha abbassato il tasso di interesse di riferimento al $0,5 \%$. Così ha fatto anche la Banca Centrale Europea che ha abbassato il tasso di riferimento sotto l'1\%.

La Banca Centrale della Repubblica di Albania non ha seguito l'esempio di queste banche e delle banche degli altri paesi in Europa e nel mondo. E 'dal 2009 e finora ha seguito questo corso: nel 2009 - 6,25\%, nel 2010 - 5\%, nel 2011 - 4,75\% , nel 2012 - 4\% e 3,75\% di oggi. Guarda quanti grandi cambiamenti ci sono. Sono proprio queste differenze che influenzano il livello dei tassi di interesse sui depositi e sui tassi di interesse attivi. Questo è un errore fatto alla Banca Centrale di Albania nei confronti del Lek in un momento in cui i suoi concorrenti hanno fatto una valutazione giusta. Questo ha stimolato la raccolta in lek e la riduzione della domanda per i prestiti in valuta nazionale. II ruolo del lek in questo modo è stato oscurato in connessione con lo sviluppo del business attraverso i prestiti per gli investimenti.

Ma dove è andato il Lek non dato sotto forma di un prestito? La quantità di tutto ciò, non dato in forma di prestito, viene aspirata attraverso la vendita di titoli di Stato e delle obbligazioni del Tesoro per lo più. È stato accordato un credito inferiore al $45 \%$ del patrimonio. II resto passa spesso per l'acquisto di titoli. Qui infatti abbiamo proprio "il fenomeno del piegamento degli investimenti privati", o il fenomeno che spesso chiamiamo Crouding Out. I soldi invece di andare in sviluppo delle imprese sono andati a coprire i costi delle spese pubbliche.

Se proviamo a collegare la dinamica dei tassi di interesse su depositi e prestiti, con la dinamica del tasso base di interesse si vedrà che non vi è alcuna connessione. Questo dimostra che la Banca Centrale non ha alcun impatto sul mercato, e di conseguenza sull'inflazione.

La Banca Centrale ha poca influenza sul tasso di interesse su depositi e prestiti della moneta lek, e non ha per niente influenza sul tasso di interesse del l'euro e del dollaro. Questi tassi vengono determinate proprio dalla BCE e il Fedi.

In questo modo la Banca d'Albania ha il controllo del tasso di interesse sulla valuta nazionale e non sulle valute estere. Ma a tenere conto della competizione, non è in grado di influenzare sulla crescita della produzione, perché le banche commerciali hanno la loro strategia di profitto che dipende in gran parte dalle politiche delle banche centrali di cui sopra. In questo modo ha perso il suo potere influente nell'economia e quindi anche nel'indicare che è il suo obiettivo principale.

Se alle banche commerciali di secondo livello sarà vietato l'accettazione di depositi in valuta estera e la concessione di prestiti in valuta estera, questo divenne un monopolio della Banca Centrale ed allora che quest'ultima inciderebbe sul mercato del credito e della raccolta che sarebbe solo nella moneta nazionale. In questo caso l'efficacia delle sue politiche sarebbe molto più evidente di quanto non sia oggi.

\subsection{In Albania non esiste nessuna banca commerciale con capitale sociale completamente statale.}

La Banca d'Albania non può controllare l'inflazione. La prima ragione è perché in Albania circolano l'euro e il dollaro apparte il Lek albanese, la cui analisi l'abbiamo fatta sul primo punto. II secondo motivo è perché non esiste alcuna banca con capitale sociale interamente statale. Proprio questo è il problema che cercheremo di chiarire in questo punto. Come incide questo fatto sulla Banca centrale per raggiungere il suo obiettivo principale che è quello di "raggiungere $e$ mantenere la stabilità dei prezzi." È stato giusto la privatizzazione totale del sistema bancario? Se non lo è stato, allora perché è sucesso?

In Albania non esiste nessuna banca commerciale con capitale sociale completamente statale. Con l'assenza delle banche commerciale con capitale interamente statale, quando c'è la circolazione libera dei depositi e di prestiti in euro e dollaro, per la Banca centrale è stato molto difficile influenzare il mercato delle valute, nonché anche una serie di indicatori o aggregati monetari. Quindi dall'analisi che abbiamo fatto non c'è stato alcun collegamento tra i tassi di interesse di base, il PIL ed altri aggregati monetari. 
Ci sono due collegamenti che non sono cambiati come in assoluto e in termini relativi, e il legame tra La Base Monetaria e il PIL e il legame tra La Base Monetaria e Aggregato $\mathrm{M}_{1}$, mentre gli altri collegamenti sono molto cambiati. I legami tra gli aggregati monetari e il tassi di inflazione sono (20116):

$\begin{array}{lr}\text { Base monetaria / Inflazione } & -0.24 \\ \text { Valuta fuori banca / Inflazione } & -0.99 \\ \text { Aggregate M1/Inflazione } & -0.68 \\ \text { Aggregate M2/Inflazione } & 0,26 \\ \text { Aggregate M3/Inflazione } & 0.33\end{array}$

I dati mostrano chiaramente che non ci sta un forte legame tra gli aggregati monetari e il tasso di inflazione. Con tutti e tre gli aggregati il collegamento è negativo, che significa che la banca applica una politica di inflazione di espansione ed l'inflazione diminuisce invece di aumentare. E 'questo fatto, e molti altri fatti che ci hanno portato alla conclusione che la Banca Centrale di Albania non può controllare l'inflazione.

Se si analizza la relazione tra tassi di cambio e i tassi di inflazione si vedrà che la relazione tra cambio di valuta lek/euro e il tasso di inflazione è negativo, mentre è positivo quello lek/dollaro. Questa relazione si osserva negli anni 2008 - 2012 per ogni mese, quindi non è solo tendenza espansiva in anni, ma anche nei mesi dello stesso anno. Ci sono particolari anni nei quali questo tasso è molto più alto.

Che cosa indicano queste relazioni? Queste relazioni indicano che quando il Lek albanese viene svalutato si riducono le importazioni e aumenta la produzione nazionale che di conseguenza provoca un aumento dell'inflazione. II contrario è accaduto al dollaro.

La relazione tra l'inflazione e i tassi di cambio ${ }^{7}$ :

Lek / EUR in relazione all'inflazione $\quad-0,26$

Lek / Dollaro in relazione all'inflazione $\quad-0,42$

Questi relazioni ci sono ogni anno, dal 2008 fino al 2012, il che significa che questa è la tendenza generale.

Questi e altri indicatori mostrano che le banche commerciali non associano le azioni della Banca centrale nella caduta del taso di interesse per conto dei depositi - prestiti, ma soprattutto guardano i loro interessi ristretti e strategici. Essi operano poco con il lek e molto di più con le valute, in modo da avere quasi una completa indipendenza da esse. Espressione di questa indipendenza sono le frequenti chiamate dal governatore della Banca Centrale ed il Primo Ministro $o$ il ministro delle Finanze a queste banche per aumentare i prestiti alle imprese. Infine si sono prese una serie di misure per agevolare i prestiti alle banche commerciali in esecuzione dei colaterali.

Lasciando libere le banche commerciali sui depositi e i prestiti in valuta estera, ha perso il suo ruolo nell'influenzare sui tassi di cambio. I tassi di cambio vengono decisi per lo più dalle banche commerciali, mentre BC è spesso vittima delle loro azioni. Se la valuta fosse un suo monopolio molti fenomeni negativi sarebbero state eliminate e l'economia poteva respirare più liberamente. Attualmente il Lek albanese ha avuto una nuova svalutazione raggiungendo 141lek a 1Euro. Questa svalutazione tende ad aumentare. La Banca del'Albania non può proteggere il Lek al significativo valutazione della valuta estera.

La Banca Centrale di Albania non ha un obiettivo diverso da "il raggiungimento e il mantenimento della stabilità dei prezzi". Un tale obiettivo è anche dei paesi europei, ma se notiamo con attenzione ci sono anche altri obiettivi che sono associati alla crescita economica ed alla diminuzione della disoccupazione. In Albania non ci sono né la stabilità sul tasso di cambio della valuta né la crescita economica o la riduzione della disoccupazione. In termini di stabilità dei tassi di cambio e l'intervento della banca centrale, vale la pena ricordare ancora una volta che queste sono azioni non riuscite. Queste azioni sarebbero efficaci se la valuta fosse monopolio della banca centrale, e non di circolare liberamente come il Lek albanese. La banca centrale non possono pregiudicare il contenimento di questo fenomeno e neanche il suo impatto sul tasso di cambio che sta diventando giorno per giorno molto inquietante.

Per gli altri paesi la valutazione del tasso di cambio è molto importante e viene visto in funzione della crescita economica. Si potrebbe dire che questi paesi operano a tassi fissi. Direi che per la protezione della moneta nazionale non è importante il cambio se il tasso di cambio è un monopolio della Banca centrale.

Per concretizzare prenderemo in esempio due paesi come l'Asia e la Repubblica popolare di Cina come un paese in via di sviluppo e l' Hong Kong della Cina come un paese molto sviluppato, che prendono di mira la conservazione

\footnotetext{
6 I dati sono tratti dalla monografia "La recessione globale ed il suo impatto sull'economia albanese". Pagina 427. Alqi Naqellari, Robert Maliqi \& Almarin Naqellari. Tirana 2012.

7 I dati sono tratti dalla monografia "La recessione globale ed il suo impatto sull'economia albanese". Pagina 430. Alqi Naqellari, Robert Maliqi \& Almarin Naqellari. Tirana 2012.
} 
della valuta nazionale. La principale autorità nel settore bancario è la Banca Popolare Cinese $\left(\mathrm{RPC}^{8}\right)$ che ha per obiettivo "il valore della valuta". L'obiettivo della politica monetaria è quello di mantenere la stabilità del valore della moneta e, quindi, promuovere la crescita economica. Come si vede, la Cina, in quanto paese in via di sviluppo non ha come obiettivo il contenimento dell'inflazione, ma di raggiungere la crescita economica attraverso la stabilizzazione del tasso di cambio dello yuan.

In Hong Kong l'obiettivo principale della autorità monetaria è "la stabilità del tasso di cambio". L'obiettivo primario della politica monetaria della autorità monetaria di Hong Kong è quello di mantenere la stabilità del tasso di cambio. Per quanto riguarda il tasso di inflazione è alto su tutti e due i paesi. Così come è alta la crescita economica nella Repubblica di Cina, è più alto anche il tasso di inflazione. Tutto l'incontrario accade in un paese come l'Albania, che in primo piano non dovrebbe avere la stabilità dei prezzi ma il tasso di cambio in funzione della crescita economica e della diminuzione del tasso di disoccupazione.

\section{La privatizzazione ed il fenomeno dell'oligopolio.}

II sistema bancario di oggi consiste solo nelle banche commerciali con capitale sociale interamente privato ed in generale straniero e nessuna banca con la capitale statale. Questa situazione ha indotto il sistema bancario nel creare una struttura di mercato di oligopolio con un primario istituto di credito del tutto straniero che è Raiffeisen Bank. La crisi finanziaria del 2008 ha evidenziato l'impatto delle banche estere nel processo della trasmissione della politica monetaria nei paesi con economie in via di sviluppo. Sono stati frequenti i casi in cui le banche centrali nei paesi con mercati emergenti, dove le banche estere hanno un ruolo dominante, hanno abbassato il tasso di interesse di riferimento, che non è stato associato ad una riduzione dei tassi di prestito.

Tali questioni sono di particolare importanza per i paesi con economie in via di sviluppo, i quali non erano la causa della crisi finanziaria del 2008, ma erano quelli che hanno sofferto particolarmente il nazionalismo finanziario che è stato innescato dalla crisi ${ }^{9}$.

L'attuale crisi fra l'altro ha evidenziato il legame tra la presenza delle banche estere e l'efficacia della politica monetaria. Se le banche straniere sono importanti operatori finanziari per l'economia nazionale e se la loro attività di finanziatore e debitore è più influenzati dalle condizioni economiche del paese madre che dalle condizioni del paese ospitante, allora l'efficacia dell'attuazione della politica monetaria è debole.

La Banca Centrale ha preso una serie di misure nel 2008, ma il loro effetto non ha confermato le aspettative, nonostante la situazione va esaminata sotto diversi punti di vista. II governatore della Banca Centrale ${ }^{10}$, ha detto che. "II credito ha rallentato bruscamente, il tasso di cambio ha cominciato a deprezzarsi e le previsioni per la crescita economica e l'inflazione è diminuita. Nel frattempo, i prelievi dei depositi sono continuati e i problemi di liquidità sono più profondi." " Prima della crisi, la Banca Centrale vedeva con preoccupazione le possibili minacce finanziarie che si accumulavano negli anni, della rapida intermediazione finanziaria. Per illustrare più specificamente: la maggior parte della crescita del credito è andato al settore non-negoziabile dell'economia, dove i servizi e le costruzioni hanno rappresentato per la maggior parte questo aumento. Inoltre, più del $70 \%$ del totale de prestiti era in valuta estera, dove si rappresentava l'euro come la quota maggiore dei finanziamenti in essere."

La Privatizzazione totale del sistema bancario ha sostituito una forma centralizzata di governo ad un altro, ma che non è soggetto dello stato. Questa sostituzione di proprietà non ha rimosso ed eliminato il fenomeno, ma ad oggi è più pronunciato l'effetto dell'oligopolio sull'indice dei prezzi. Oggi le due banche più grandi hanno un patrimonio di 46,92\% e $39,84 \%$ di depositi. Le tre banche più grandi rappresentano il $57,6 \%$ del patrimonio e il $48,8 \%$ del conto di deposito. Guardate che concentriazione c'è sul sistema bancario in cui operano 16 banche. Queste banche costituiscono un oligopolio bancario e determinano i prezzi di mercato. Essi creano l'assenza della concorrenza nel mercato e l'evitare di seguire l'attuazione della politica della banca centrale. Se non vi era stato la privatizzazione dell' ex Cassa di risparmio, essendo di diritto il braccio destro della Banca centra avrebbe seguito le sue politiche in materia. In questo modo avremmo una forte concorrenza sul mercato. Non ci sarebberò tutte le tendenze negative che abbiamo oggi.

L'effetto della privatizzazione totale non era solo nelle aree di cui abbiamo parlato, ma anche in molte altre.

\footnotetext{
${ }^{8}$ Fonte: Adattato da Genberg e Lui (2009)

${ }^{9}$ Meir Sokoler. Relazione tra stabilità monetaria e finanziaria - Insegnamenti per le economie in via di sviluppo. Meir Sokoler, Consigliere del Fondo monetario internazionale per la politica monetaria della Banca d'Albania.

1014 dic 2010. La stabilità macro-finanziaria nei paesi candidati e potenziali candidati verso I'UE: lezioni da imparare ", Francoforte, 14 Dicembre 2010. 


\section{Altre conseguenze della riorganizzazione del sistema bancario.}

L'economia albanese nel 2008 ha visto il più alto tasso di crescita economica, non solo, ma quasi tutti gli indicatori macroeconomici sono stati su livelli soddisfacenti. L'indicatore negativo è stato solo un debito pubblico aumentato visibilmente. Questa tendenza non è continuata in futuro.

Alla seconda metà del 2008 ed in seguito, l'economia albanese stava affrontando la recessione e la crisi finanziaria globale. La Banca Centrale con le sue politiche monetarie tendeva ad attenuare l'impatto negativo della pressione esterna sull'economia albanese. Essa non raggiunse mai questo obiettivo per le ragioni che abbiamo dato a questo lavoro, ma anche perchè non è riuscita a coordinare la politica fiscale con quella monetaria. La crescitaeconomica dal 2009 al 2012 è in calo, nonostante gli indicatori monetari sono rimasti stabili oppure sono diminuiti. Dove sta riflettendo l'impatto della riorganizzazione del sistema bancario?

Le aree della nostra analisi sono:

- In primo luogo, la distribuzione dei prestiti tra settori di produzione, nell'industria, nell'agricoltura, nei servizi, nel turismo, ecc. La privatizzazione delle banche commerciali ha avuto un impatto negativo sui prestiti nei settori più importanti dell'economia, come l'agricoltura, l'idustria dell'estrazione e della lavorazione , il turismo, ecc. II prestito è più concentrato nei settori che sono privi di rischi e nei settori gestiti dalle società straniere e le società miste come il commercio, l'edilizia, l'energia, l'industria di trasformazione, ecc.

- In secondo luogo, la distribuzione dei depositi e dei prestiti in valuta nazionale ed estera. I depositi sono in crescita ma a ritmi più elevati rispetto al livello dell crescita economica. La loro struttura è cambiata in favore della valuta estera a causa della sfiducia del lek albanese e del suo continuo deprezzamento. Ciò ha portato al risultato che sia i prestiti siano su tali strutture. I prestiti in valuta estera colpisce di più sulla crescita dell'economia.

- In terzo luogo, la differenza tra i depositi e i prestiti per tipo di valuta. Da i depositi in lek con o senza termine al massimo il $50 \%$ di loro sono concessi ai soggetti economici sotto forma di un prestito, il resto viene utilizzato per l'acquisto dei strumenti del debito pubblico, mentre il $100 \%$ dei depositi in valuta estera concessi come prestito, indipendentemente alle aziende nazionali o estere.

- In quarto luogo, la distribuzione dei prestiti a seconda dei soggetti economici. La struttura dei finanziamenti a seconda di enti e valute è quasi la stessa. L'azienda utilizza la valuta per l'acquisto di macchinari e attrezzature, per lo più per le importazioni, mentre gli individui lo utilizzano in genere per effettuare pagamenti all'interno del paese per gli appartamenti, auto, ecc.

- In quinto luogo, la distribuzione dei crediti per territorio, ecc. La distribuzione di credito in genere mostra che l'economia albanese non è basata sul livello appropriato di credito da parte delle banche commerciali, creando sproporzioni di sviluppo tra i diversi distretti.

\section{La conclusione generale:}

- L'inflazione è generalmente importata e non può essere controllata dalla Banca Centrale. Essa può influire ma non può contollare.

- La libera circolazione dell'euro e del dollaro ha dato conseguenze negative sull'economia, riducendo il ruolo del lek e della politica monetaria Banca centrale. Per questo è necessario la valuta estera sia considerata come l'oro e deve essere il suo monopolio.

- La privatizzazione delle banche commerciali non ha avuto effetti positivi sulla crescita economica e l'attuazione della politica monetaria della Banca Centrale. Per questo è necessaria la nascità della Banca Commerciale con capitale sociale interamente statale.

\section{Bibliografia}

Rapporto statistico. Banca d'Albania. 03/2013. Tirana

Rapporto statistico. Banca d'Albania. 10/2012. Tirana

Annuario statistico. Ministero delle Politiche Agricole nel 2009

Annuario statistico della Albania (Annuario Statistico di Albania) 1991. Ministero dell'Economia. Dipartimento di Statistica.

Rapporto di politica monetaria per il terzo trimestre del 2012. Banca d'Albania 
"La recessione globale ed il suo impatto sull'economia albanese". Monografia. Alqi Naqellari Robert Maliqi \& Almarin Naqellari Tirana 2012.

Conferenza Internazionale di I. Alba Scienze di Skopje 29-31 Agosto 2012. "Nuovo Modello economico volto pressione della recessione globale" A.Naqellari, T.Mehilli \& Jeton Shaqiri.

Conferenza internazionale Scientifica. L'Albania e la crisi: Quali sono le prospettive? Rivisitare il modello di crescita. 15-16 dicembre 2012. "Effetti della politica monetaria sull'economia durante la recessione globale 2008-2011". Alqi Naqellari \& Terida Mehilli

La quinta conferenza scientifica internazionale "Gli investimenti in funzione dello sviluppo economico e il processo di integrazione". Ulqin, aprile 2013. "Il Sistema finanziario e il suo impatto sull' economia albanese". Alqi Naqellari \& Guximtar Rushani.

L'Albania in cifre. 2010, INSTAT 